\title{
Mise en œuvre de schémas numériques pour l'étude d'écoulements diphasiques instationnaires
}

\author{
Michel Barret $^{1, a}$, Éric Faucher ${ }^{2}$, Jean-Marc Hérard ${ }^{3}$ et Jean-FÉlix Durastanti ${ }^{1}$ \\ 1 Université Paris Val-de-Marne - IUT de Sénart, Avenue Pierre Point, 77127 Lieusaint, France \\ 2 Direction des Études et Recherches EDF - Département MTC, Route de Sens - Ecuelles, 77818 Moret-sur-loing Cedex, France \\ 3 Direction des Études et Recherches EDF - Département MFTT, 6 quai Wattier, 78400 Chatou, France
}

Reçu le 28 mars 2003, accepté le 2 juillet 2004

\begin{abstract}
Résumé - Cet article propose la mise en œuvre de schémas numériques permettant le calcul d'écoulements diphasiques liquide-vapeur monodimensionnels instationnaires. Les cas tests classiques sont décrits avec leur étude de convergence. Des comparaisons avec des résultats expérimentaux stationnaires sont aussi données.
\end{abstract}

Mots clés : Méthode des volumes finis / solveur de Roe / modèle homogène relaxé / écoulements autovaporisants

\begin{abstract}
On the implementation of numerical schemes for the study of unsteady two-phase flows. This paper deals with the application of numerical schemes to the calculation of two-phase flows. Some classical one-dimensional flows are tested with those numerical schemes. Comparisons with experimental two-phase flows are also given.
\end{abstract}

Key words: Finite volume method / Roe's solver / Homogeneous Relaxation Model / Flashing flow

\section{Introduction}

Ce travail s'inscrit dans le cadre de la simulation des écoulements diphasiques eau-vapeur à travers les organes de robinetterie industrielle, tels que soupapes de sûreté ou disques de rupture. Ces composants sont utilisés pour la protection des circuits de centrales nucléaires contre les surpressions accidentelles.

Le problème consiste à savoir modéliser l'écoulement instationnaire d'un liquide qui, par suite d'une dépressurisation, va se vaporiser au passage de l'organe de détente. Nous avons orienté notre recherche selon deux directions parallèles. La première nécessite de savoir représenter correctement le retard au changement de phase qui est la conséquence d'une chute rapide de pression au sein du liquide.

La seconde met en œuvre des schémas numériques «volumes finis » $[1-3]$ qui soient capables de reproduire les paramètres du fluide en écoulement, sachant que le nombre de Mach varie de quelques dixièmes à plusieurs unités avec formation d'ondes de chocs de condensation.

Nous décrirons tout d'abord dans cet article les deux modèles physiques que nous avons retenus pour représenter le retard au changement de phase.

\footnotetext{
a Auteur correspondant : barret@univ-paris12.fr
}

Nous décrirons ensuite le solveur VFRoe [4] en variables non conservatives (VFRoe-ncv) et le solveur de Rusanov.

L'application de ces deux schémas au calcul d'un écoulement en tuyère sera ensuite montrée et le résultat de ces calculs en écoulement stationnaire sera commenté.

\section{Les modèles de changement de phase instationnaire}

Les équations d'évolution pour les écoulements autovaporisants ont été obtenues en supposant l'égalité des pressions et des vitesses dans les deux phases, et en considérant que la vapeur est saturée. Elles sont données ci-dessous :

$$
\begin{aligned}
& \frac{\partial}{\partial t}(A \rho)+\frac{\partial}{\partial z}(A \rho u)=0 \\
& \frac{\partial}{\partial t}(A \rho x)+\frac{\partial}{\partial z}(A \rho u x)=A \Gamma \\
& \frac{\partial}{\partial t}(A \rho u)+\frac{\partial}{\partial z}\left[A \rho u^{2}\right]+A \frac{\partial P}{\partial z}=-A f-A \rho g \cos (\varphi) \\
& \frac{\partial}{\partial t} A E+\frac{\partial}{\partial z}[A(E+P) u]+P \frac{\partial A}{\partial t}=-A \rho g \cos (\varphi) u \\
& +A Q-A Q_{\mathrm{frot}}
\end{aligned}
$$




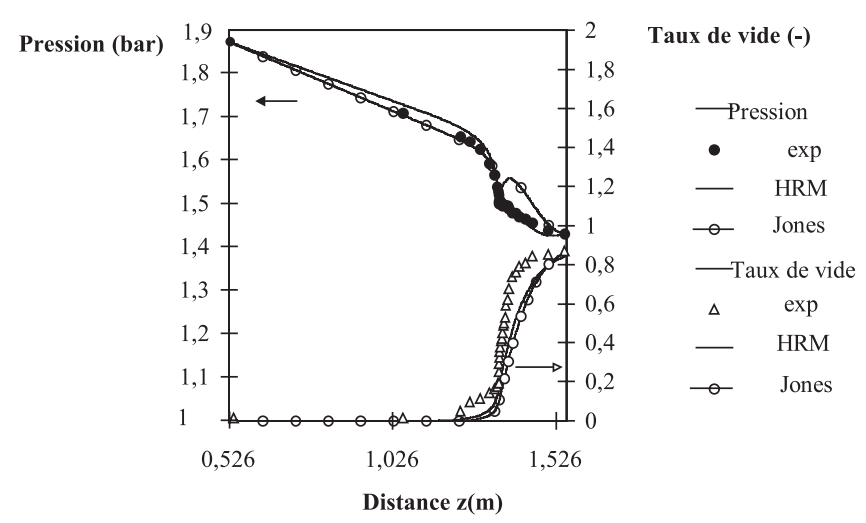

Fig. 1. Courbes de pression et de taux de vide pour l'essai $\mathrm{n}^{\circ} 405$ de Moby-Dick [8]. Débit ${ }_{\exp }=2,74 \mathrm{~kg} \cdot \mathrm{s}^{-1}$, débit $_{\mathrm{HRM}}=$ $2,61 \mathrm{~kg} . \mathrm{s}^{-1}$, débit Jones $=2,75 \mathrm{~kg} . \mathrm{s}^{-1}$.

équations dans lesquelles $z$ est l'abscisse, $t$ le temps, $A$ est la section droite, $u$ la vitesse moyenne, $\rho$ la masse volumique, $x$ le titre, $P$ la pression, $E$ l'énergie totale, $\Gamma$ le terme de transfert de masse entre les deux phases, $Q$ la chaleur échangée avec l'extérieur et $Q_{\text {frot }}$ l'énergie perdue en frottement.

Il s'agit maintenant de préciser les expressions pour les termes sources, transfert de masse entre les phases, le frottement pariétal et les transferts de chaleur à la paroi. Pour cela, deux modèles physiques ont été retenus : le modèle homogène relaxé (HRM) proposé par Bilicki et al. [5-7] et le modèle de nucléation à la paroi proposé par Jones et al. [8-10].

Une comparaison de ces deux modèles a été effectuée en comparaison avec des écoulements stationnaires fournis par Réocreux [11] (voir Fig. 1).

Dans l'ensemble, l'utilisation du modèle HRM et du modèle de Jones et al. pour les écoulements en autovaporisation dans une tuyère donne des résultats satisfaisants. La corrélation du temps de relaxation apparaissant dans le modèle HRM n'est valable que pour des pressions inférieures à 25 bars. En effet, même si elle permet d'estimer correctement le débit pour des pressions plus élevées, les évolutions de la pression et du taux de vide ne sont par contre pas très bonnes.

Pour sa part, le modèle de Jones et al., qui s'appuie sur des hypothèses physiques plus solides, reste performant sur une grande gamme de pression. En effet, il commet toujours la même erreur, c'est-à-dire qu'il sous-estime la perte de charge lors de l'apparition des premières bulles de vapeur, ce qui entraîne une surestimation systématique du débit. De plus, en tenant compte de la structure de l'écoulement, il arrive à prendre en compte l'effet de décollement dans le divergent.

Ces deux modèles ont donc leurs avantages et leurs inconvénients. Celui de Jones et al. peut être utilisé directement pour des calculs dans de nouvelles géométries et avec des conditions thermodynamiques différentes, sans trop perdre de précision. Malheureusement sa complexité rend le développement de nouvelles corrélations très difficile. Si les résultats du modèle HRM actuel sont moins

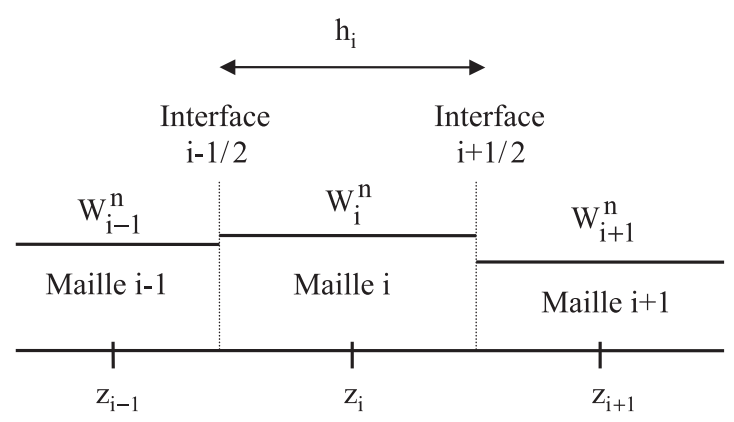

Fig. 2. Maillage unidimensionnel.

bons, sa simplicité facilite sa mise en œuvre dans le cas d'un écoulement instationnaire.

De plus, le développement d'une nouvelle corrélation pour le temps de relaxation, adaptée aux pressions supérieures à 80 bars, est possible en utilisant la méthode proposée par Bilicki et al. [5]. Mais il n'y a aucun moyen d'assurer qu'elle sera encore valable dans une autre géométrie.

\section{Les schémas numériques « volumes finis »}

Cette partie présente quelques schémas numériques pour la résolution d'un système de lois de conservation :

$$
W_{, t}+F(W)_{, z}=0
$$

$W$ représente le vecteur ayant pour composantes les variables d'état du système; par exemple, dans le cas du modèle d'écoulement diphasique proposé à la section 1 , ce vecteur $W$ a pour composantes $A, \rho . A . x, \rho . A, \rho . A . u$ et A.E.

Dans ce qui suit, $W_{\mathrm{L}}$ et $W_{\mathrm{R}}$ désignent les valeurs respectivement à gauche et à droite de l'interface $i+1 / 2$. Dans le cas des schémas explicites d'ordre 1 , on a $W_{\mathrm{L}}=$ $W_{i}^{n}$ et $W_{\mathrm{R}}=W_{i+1}^{n}$ (Fig. 2).

\subsection{Le schéma de Rusanov [12]}

C'est un schéma volumes finis qui s'écrit :

$$
W_{i}^{n+1}=W_{i}^{n}-\frac{(\Delta t)^{n}}{h_{i}}\left[G_{i+1 / 2}^{\text {Rusanov }}\left(W^{n}\right)-G_{i-1 / 2}^{\text {Rusanov }}\left(W^{n}\right)\right]
$$

Le calcul du flux numérique de Rusanov à l'interface ne demande pas de résoudre de problème de Riemann. Il s'écrit simplement :

$$
\begin{aligned}
G_{i+1 / 2}^{\text {Rusanov }}\left(W^{n}\right)=\frac{1}{2}\left[F\left(W_{\mathrm{L}}\right)+F\left(W_{\mathrm{R}}\right)\right. & \\
& \left.-s_{i+1 / 2}\left(W_{\mathrm{R}}-W_{\mathrm{L}}\right)\right]
\end{aligned}
$$

où :

$$
s_{i+1 / 2}=\max _{k}\left[\left|\lambda_{k}\left(W_{\mathrm{L}}\right)\right|,\left|\lambda_{k}\left(W_{\mathrm{R}}\right)\right|\right]
$$

$\lambda_{k}$ étant les valeurs propres de la matrice jacobienne $F^{\prime}(W)$. 
Ce schéma consistant est très peu coûteux en temps de calcul et présente l'avantage d'être utilisable pour tout système conservatif hyperbolique, en particulier pour le cas qui nous intéresse des équations d'Euler pour un fluide réel.

De plus, ce schéma est très stable et assure lui aussi de conserver la positivité de la masse volumique. Cependant, comme nous le verrons par la suite, ce schéma pèche par sa précision. Ceci est surtout pénalisant pour les écoulements multidimensionnels où les contraintes de temps de calcul limitent l'utilisation de maillages très fins.

\subsection{Le schéma de VFRoe-ncv [13]}

Ce schéma est aussi un schéma volumes finis :

$$
\begin{aligned}
W_{i}^{n+1}=W_{i}^{n}-\frac{(\Delta t)^{n}}{h_{i}}\left[G_{i+1 / 2}^{\mathrm{VFROE-ncv}}(\right. & \left.W^{n}\right) \\
& \left.-G_{i-1 / 2}^{\mathrm{VFROECv}}\left(W^{n}\right)\right]
\end{aligned}
$$

Pour calculer le flux numérique, on introduit d'abord une nouvelle variable d'état $Y$, et on effectue le changement de variable $W \rightarrow Y(W)$. Le système continu (4.1) devient :

$$
W(Y)_{, t}+F[W(Y)]_{, z}=0
$$

soit pour les solutions régulières :

$$
Y_{, t}+\left[W_{, Y}(Y)\right]^{-1}\left[F_{, W}[W(Y)]\right]\left[W_{, Y}(Y)\right] Y_{, z}=0
$$

ou encore :

$$
Y_{, t}+B(Y) Y_{, z}=0
$$

On associe donc le problème hyperbolique linéaire suivant :

$$
Y_{, t}+B(\bar{Y}) Y_{, z}=0
$$

où $\bar{Y}=\left(Y_{\mathrm{L}}+Y_{\mathrm{R}}\right) / 2$, avec comme conditions initiales :

$$
\left\{\begin{array}{l}
Y(z<0, t=0)=Y_{\mathrm{L}}=Y\left(W_{\mathrm{L}}\right) \\
Y(z>0, t=0)=Y_{\mathrm{R}}=Y\left(W_{\mathrm{R}}\right)
\end{array}\right.
$$

Ce problème de Riemann linéaire se résout très simplement. La solution se compose de $n$ états intermédiaires constants séparés par des discontinuités de contact. Les valeurs de ces états se calculent de la manière suivante : la différence entre les états initiaux $Y_{\mathrm{R}}$ et $Y_{\mathrm{L}}$ est décomposée sur la base des vecteurs propres à droite de $B(\bar{Y})$

$$
Y_{\mathrm{R}}-Y_{\mathrm{L}}=\sum_{i=1}^{n} \alpha_{i} r_{i}
$$

Les coefficients $\alpha_{i}$ sont donnés par la relation :

$$
\alpha_{i}=\left(l_{i}\right)^{t}\left(Y_{\mathrm{R}}-Y_{\mathrm{L}}\right)
$$

où les $l_{i}$ sont les vecteurs propres à gauche de $B(\bar{Y})$. Les états intermédiaires sont alors calculés par :

$$
Y_{k}-Y_{\mathrm{L}}=\sum_{i=1}^{k} \alpha_{i} r_{i}
$$

La figure 3 donne une représentation schématique de cette solution.

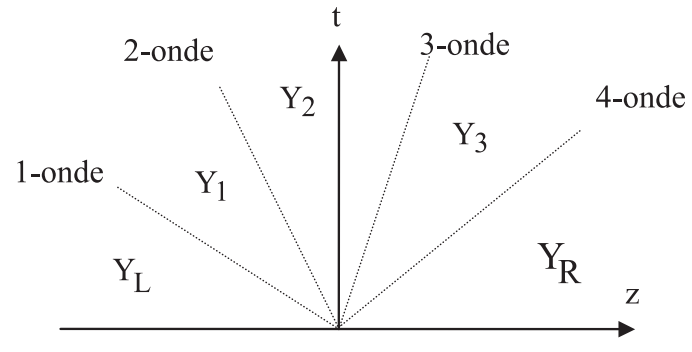

Fig. 3. Calcul des états intermédiaires.

\section{Remarque}

Il n'existe pas de justification précise pour le choix de la variable d'état $Y$. Certaines conditions doivent néanmoins être remplies [13]. Pour le modèle HRM en gaz parfait, $Y$ a pour composantes $(x, \tau, u, P)^{t}$.

\section{Cas tests instationnaires}

Plusieurs cas tests ont été étudiés, c'est-à-dire comparés avec des résultats de référence. Ces derniers peuvent être les solutions analytiques, lorsqu'elles existent, ou bien des résultats de calcul sur 100000 mailles. Les courbes de convergence ont été ainsi obtenues [14].

Nous ne donnerons ici que les résultats obtenus sur une tuyère en écoulement stationnaire (l'état stationnaire étant la limite asymptotique temporelle donnée par le schéma instationnaire).

La section de la tuyère convergente divergente est donnée en fonction de l'abscisse $z$ par l'expression (Fig. 4) :

$$
\begin{cases}A(z)=4 & \text { si } 0<z<1 / 6 \\ A(z)=-54 z^{2}+18 z+2,5 & \text { si } 1 / 6<z<1 / 3 \\ A(z)=54 z^{2}-54 z+14,5 & \text { si } 1 / 3<z<2 / 3 \\ A(z)=-54 z^{2}+90 z-33,5 & \text { si } 2 / 3<z<5 / 6 \\ A(z)=4 & \text { si } 5 / 6<z<1\end{cases}
$$

Les conditions thermodynamiques en entrée sont fixées à : $P_{\mathrm{E}}=15$ bar, enthalpie $h_{\mathrm{E}}=860 \mathrm{~kJ} . \mathrm{kg}^{-1}$, et $x=\bar{x}\left(P_{\mathrm{E}}, h_{\mathrm{E}}\right)$ tandis qu'en sortie, on impose la pression $P_{\mathrm{S}}=10$ bar.

Comme pour les autres cas tests de ce type, le mélange est initialement supposé au repos dans les mêmes conditions thermodynamiques que celles imposées en entrée.

Les résultats des trois schémas (Rusanov, VFRoencv et Roe modifié) sur 1000 mailles sont donnés sur la figure 5 .

La première constatation est que les schémas de Rusanov et de Roe modifié ne permettent absolument pas d'obtenir une solution correcte. Alors qu'en utilisant le schéma VFRoe-ncv, l'écoulement est critique et un choc est présent dans le divergent, les résultats des deux autres schémas ne contiennent même pas de point sonique.

Considérant uniquement les résultats du schéma VFRoe-ncv, on constate que le défaut lié à la variation de section dans le cas de l'eau liquide disparaît pour un 


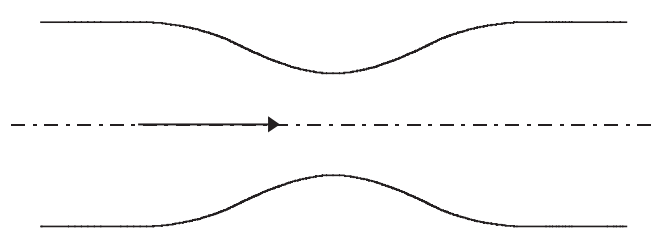

Fig. 4. Schéma de la tuyère convergente divergente.
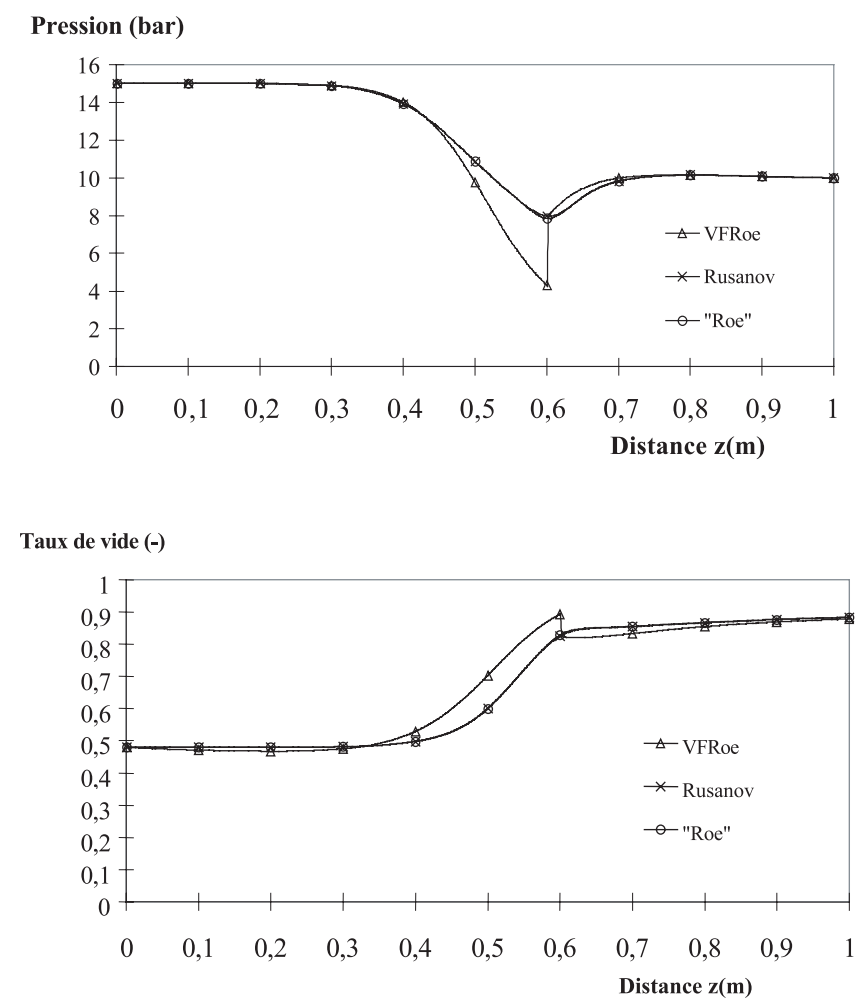

Fig. 5. Écoulement diphasique stationnaire en tuyère. Résultats donnés par les schémas numériques (1000 mailles).

écoulement diphasique, ce qui confirme qu'il était dû à la très faible variation de la masse volumique avec la pression. De plus, la figure 5 confirme que le taux de vide varie au passage du choc stationnaire, puisque la pression et la masse volumique varient à travers le choc, alors que le titre reste constant.

\section{Conclusion}

Le travail que nous avons entrepris a mis en évidence que le schéma VFRoe en variables non conservatives est le plus précis. Pour des écoulements instationnaires, les trois schémas sont à peu près équivalents, mais dans le cas stationnaire, le schéma de Rusanov et le schéma de Roe modifié sont nettement moins précis.

Cette différence est particulièrement marquée dans le cas des écoulements liquides ou diphasiques, où il est nécessaire avec ces deux schémas d'utiliser des maillages plus de dix fois plus fins, pour obtenir une précision comparable à celle du schéma VFRoe-ncv.

On peut se demander si l'origine des bonnes performances du schéma VFRoe-ncv est liée au fait qu'il appartient à la classe Godunov, c'est-à-dire qu'il utilise le flux analytique pris en un état approché, contrairement au schéma de Rusanov et au schéma de Roe modifié, ou si cela est lié à l'utilisation des variables $(1 / \rho, u, P)$ pour le problème linéarisé, dans la mesure où la variation relative de la variable conservative $W$ est peu sensible à la variation relative de $P$, qui est l'une des variables « motrices » du problème de Riemann non linéaire unidimensionnel.

Néanmoins, le schéma de Rusanov reste intéressant, car il est nettement plus robuste que les deux autres. Une stratégie industrielle a donc été mise en place : on utilise prioritairement le schéma VFRoe-ncv, et pour les cas de démarrages violents, on débute le calcul avec le schéma de Rusanov, avant de basculer sur le schéma VFRoe-ncv une fois les effets non linéaires partiellement établis (ondes formées).

\section{Références}

[1] R. Saurel, M. Larini, J.C. Loraud, Exact and approximate Riemann solver for real gases, J. Comp. Phys. 112(1) (1994a) 126-137

[2] R. Saurel, E. Daniel, J.C. Loraud, Two-phase flows: second-order schemes and boundary conditions, AIAA J. 32(6) (1994b) 1214-1221

[3] E. Toro, Riemann solvers and numerical methods for fluid dynamics, Springer Verlag, 1997

[4] P.L. Roe, Approximate Riemann solvers, parameter vectors and difference schemes, J. Comp. Phys. 43 (1981) $357-372$

[5] Z. Bilicki, J. Kestin, M.M. Pratt, A reinterpretation of the results of the Moby-Dick experiments in terms of the nonequilibrium model, J. Fluid Engng. 112 (1990) 212-217

[6] P. Downar-Zapolski, Z. Bilicki, L. Bolle, J. Franco, The non-equilibrium model for one-dimensionnal flashing liquid flow, Int. J. Multiphase Flow 22 (1996) 473-483

[7] Z. Bilicki, J. Kestin, Physical aspects of the relaxation model in two phase flow, Proc. R. Soc. Lond. A428, 1990, pp. 379-397

[8] T.S. Shin, O.C. Jones, Nucleation and flashing in nozzles1: a distributed nucleation model, Int. J. Multiphase Flow 19 (1993) 943-964

[9] V.N. Blinkov, O.C. Jones, B.I. Nigmatulin, Nucleation and flashing in nozzles-2: comparison with experiments using a five-equation model for vapour void development, Int. J. Multiphase Flow 19 (1993) 965-986

[10] T.S. Shin, O.C. Jones, An active cavity model for flashing, Nuclear Engineering and Design 95 (1986) 185-196

[11] M. Réocreux, Contribution à l'étude des débits critiques en écoulement diphasique eau-vapeur, Thèse de doctorat es-sciences, Université Scientifique et Médicale de Grenoble, France, 1974

[12] V.V. Rusanov, The calculation of the interaction of nonstationary shock waves and obstacles, Zh. Vich. Nat. 2 (1961) 304-320

[13] T. Buffard, T. Gallouët, J.M. Hérard, Schéma VFROE en variables caractéristiques, Principe de base et application aux gaz réels, rapport EDF HE-41/96/041/A, 1996

[14] E. Faucher, Simulation numérique des écoulements unidimensionnels instationnaires avec autovaporisation, thèse de doctorat en systèmes énergétiques et contrôle des processus, Université Paris XII-Créteil, 2000 